\title{
Poly(e-caprolactone) Biocomposites Based on Acetylated Cellulose Fibers and Wet Compounding for Improved Mechanical Performance
}

\author{
Giada Lo Re, ${ }^{* \dagger}$ ○ Stephen Spinella, ${ }^{\ddagger}$ Assya Boujemaoui, ${ }^{\dagger}$ Fabiola Vilaseca, ${ }^{\S}$ Per Tomas Larsson, ${ }^{\dagger, \|}$ \\ Fredrik Adå," and Lars A. Berglund ${ }^{*, \dagger}$
}

${ }^{\dagger}$ Division of Biocomposites and Wallenberg Wood Science Center, School of Chemical Science and Engineering, Department of Fibre and Polymer Technology, KTH Royal Institute of Technology, Teknikringen 56, Stockholm, SE-100 44, Sweden

${ }^{\ddagger}$ NYU Tandon School of Engineering, Six Metrotech Center, Brooklyn, New York 11201, United States

${ }^{\S}$ BIMATEC Group, Department of Chemical Engineering, Agricultural and Food Technology, University of Girona, C/Maria Aurèlia Capmany 61, 17003 Girona, Spain

"RISE Bioeconomy, Teknikringen 56, Stockholm, SE-100 44, Sweden

\section{Supporting Information}

ABSTRACT: $\operatorname{Poly}(\varepsilon$-caprolactone) (PCL) is a ductile thermoplastic, which is biodegradable in the marine environment. Limitations include low strength, petroleum-based origin, and comparably high cost. Cellulose fiber reinforcement is therefore of interest although uniform fiber dispersion is a challenge. In this study, a one-step wet compounding is proposed to validate a sustainable and feasible method to improve the dispersion of the cellulose fibers in hydrophobic polymer matrix as PCL, which showed to be insensitive to the presence of the water during the processing. A comparison between unmodified and acetylated cellulosic wood fibers is made to further assess the net effect of the wet feeding and chemical modification on the biocomposites properties, and the influence of acetylation on fiber structure is

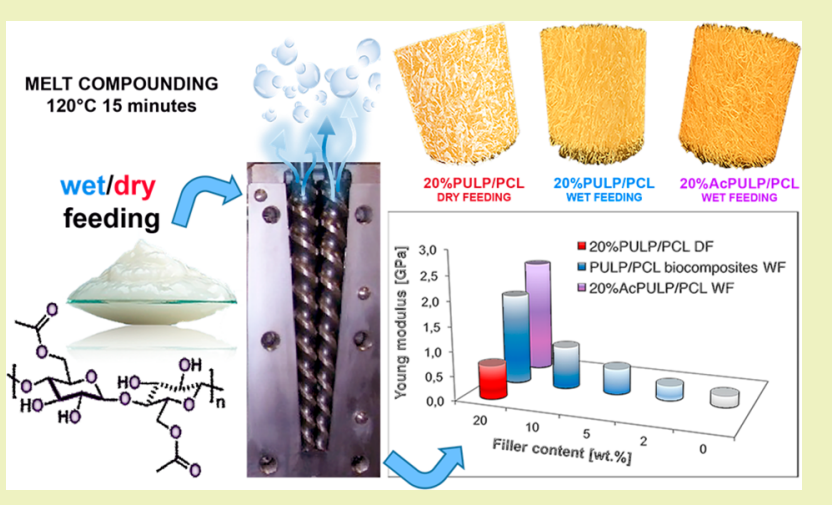
reported (ATR-FTIR, XRD). Effects of processing on nano-

fibrillation, shortening, and dispersion of the cellulose fibers are assessed as well as on PCL molar mass. Mechanical testing, dynamic mechanical thermal analysis, FE-SEM, and X-ray tomography is used to characterize composites. With the addition of 20 wt \% cellulosic fibers, the Young's modulus increased from $240 \mathrm{MPa}$ (neat PCL) to $1850 \mathrm{MPa}$ for the biocomposites produced by using the wet feeding strategy, compared to $690 \mathrm{MPa}$ showed for the biocomposites produced using dry feeling. A wet feeding of acetylated cellulosic fibers allowed even a greater increase, with an additional $46 \%$ and $248 \%$ increase of the ultimate strength and Young's modulus, when compared to wet feeding of the unmodified pulp, respectively.

KEYWORDS: Cellulose, Poly(E-caprolactone), Extrusion, Biodegradable, Biocomposite, Wet feeding, Nanofibrillation, Acetylated cellulose

\section{INTRODUCTION}

Poly ( $\varepsilon$-caprolactone) (PCL) is an important biodegradable polyester produced at the industrial scale with advantages such as high strain to failure and facile melt processing. ${ }^{1}$ However, the application field of PCL-based materials is limited by its low tensile strength (16-24 MPa), Young's Modulus (240-420 $\mathrm{MPa}$ ) and higher cost compared to polyolefins. ${ }^{2}$ PCL's low melting point (ca. $60{ }^{\circ} \mathrm{C}$ ) allows processing temperatures suitable for lignocellulosic materials so that thermal degradation associated with polypropylene (PP) and polylactide (PLA) $\left(200{ }^{\circ} \mathrm{C}\right)$ may possibly be limited.

Lignocellulosic fibers have relatively low cost, come from renewable resources and can be used as reinforcement for polymeric biocomposites, ${ }^{3-5}$ in particular for biodegradable composites. $^{6-8}$ They possess high mechanical properties, as Young's modulus of nanocrystalline cellulose is around 140-
$150 \mathrm{GPa}{ }^{9,10}$ Lignocellulosic fibers may suffer from low thermal stability at common processing temperatures. Pyrolytic degradation of the cellulosic materials can take place at temperatures above $140^{\circ} \mathrm{C}$, depending on the cellulosic source, leading to an undesirable brownish discoloration and compromising the composites performance. ${ }^{11}$ Other challenges include high moisture sorption and poor compatibility with hydrophobic polymers. The moisture sorption could be addressed by including the fibers in highly hydrophobic matrices, although the lack of compatibility may lead to poor fiber dispersion in the resulting composites.

Received: February 2, 2018

Revised: $\quad$ March 2, 2018

Published: March 27, 2018 
With the aim to produce a high strength biodegradable composite, PCL was melt blended with cellulose nanocrystals (CNCs) by in situ grafting of PCL to CNC surfaces, and improved mechanical properties were observed. ${ }^{12}$ Synthesis of CNC-g-PCL required a controlled reaction environment and time-consuming solvent exchange processing, which limits scalability. Acetylation has been considered as a scalable method practiced in industry to improve cellulose adhesion to hydrophobic matrices, ${ }^{13,14}$ and for its promising potential as a new green emerging method. ${ }^{15,16}$ Degree of substitution (DS) values below 0.48 are recommended for composite applications because higher DS values result in decreased cellulose crystallinity and degree of polymerization. ${ }^{17,18}$

In addition to compatibility issues, there are other processing challenges with cellulosic fibers related to drying. Although it seems practical to first dry cellulosic fibers prior to compounding, this can lead to substantial and irreversible fiber and fibril aggregation (e.g., hornification), ${ }^{19,20}$ that makes difficult fiber redispersion during melt compounding.

Among melt compounding techniques, a "wet feeding" (from water dispersion) strategy is used by the automotive industry for clay-based nylon or polyolefin composites, ${ }^{21-23}$ allowing for the production of high performance clay based nanocomposites containing exfoliated clay. Polylactide/CNC composites have been prepared using a "liquid-feeding" (from solvent dispersion) strategy. This resulted in reduced aggregation and increased mechanical properties compared to neat PLA. ${ }^{24}$ Beaugrand and Berzin assessed the nanofibrillation of humid sisal hemp fibers/PCL biocomposites. ${ }^{25}$ Yano et al. ${ }^{26-28}$ proposed the use of never dried pulp in the presence of different compatibilizers. The purpose was to reduce interfacial energy between pulp and polyolefins during processing and to have in situ nanofibrillation of the pulp. Improved mechanical properties were obtained due to the creation of nanocomposites based on fibrillated pulp fibers. However, the proposed processing approach suffers from cost- and timeconsuming solvent exchanges combined with multistep compounding.

The present study contributes to the validation of a one-step wet feeding method as an effective green and sustainable approach for the melt compounding of cellulose fibers/PCL biocomposites. The results demonstrate an efficient and improved dispersion of the fibers and the preservation of their length, which resulted in a significant improvement of the mechanical performance of the biocomposites produced by wet feeding. The presented approach leads the production of cellulose pulp fibers/PCL biocomposites avoiding the use of organic solvent or solvent exchange steps prior to the processing, and paves a route for a new generation of sustainable cellulose-based biocomposites produced by wet feeding during melt processing. Moreover, a comparison with acetylated pulp fibers is included in the study, to highlight the effect of the chemical modification as a potential further strategy to further improve the biocomposites, which showed effective only if coupled with the wet feeding approach. A traditional pulp fiber acetylation was used to better control the degree of the surface modification. Here, it was of interest to evaluate the potential of cellulosic fiber reinforcement in PCL biocomposites. Modulus and yield strength of PCL are quite low, and it is often observed that fiber reinforcement is not so efficient for ductile matrices. Embrittlement effects may dominate so that strength is even decreased. Here the hypothesis is that if fiber agglomeration can be avoided, the embrittlement effects can be limited and strength will be increased. A cellulosic bleached Kraft pulp was used and fiber content was varied from 0 to 20 wt $\%$.

\section{EXPERIMENT AND MATERIALS}

Materials. PCL grade Capa $6506\left(M_{\mathrm{w}}=50000 \mathrm{~g} / \mathrm{mol}\right.$, melt flow index $=11.3-5.2 \mathrm{~g} / 10 \mathrm{~min}$ with $2.16 \mathrm{~kg}, 1 \mathrm{in}$. PVC die at $160^{\circ} \mathrm{C}$ ) was kindly provided by Perstorp Holding AB, Sweden. Bleached softwood Kraft fibers from pine (K46) were supplied by SCA Forest Products (Östrand pulp mill, Timrå, Sweden). The pine pulp fibers were soaked in deionized water overnight and then dispersed with a laboratory reslusher operated at $30000 \mathrm{rpm}$ (Ultra Turrex T $25 \mathrm{D}$ IKA, Germany). They were afterward washed with acetate buffer ( $\mathrm{pH} 4.6)$ containing $2.4 \mathrm{NaClO}_{2}$ for $1 \mathrm{~h}$ at $60{ }^{\circ} \mathrm{C}$, in order to remove any impurities, and then used for a solvent exchange for the acetylation or directly filtered to the final $22 \%$ dry content in cellulosic fibers in the material used for the melt processing.

Acetyl chloride ( $\geq 99 \%$ ), triethyl amine (TEA) ( $\geq 99 \%$ ), and sodium chlorite $\left(\mathrm{NaClO}_{2}\right)$ were purchased from Sigma-Aldrich. Acetone (analytical grade) and tetrahydrofuran (THF) ( $\geq 99 \%$ ) were purchased from VWR. All reagents and solvents were used as received without further purification.

Acetylation of Pine Pulp Fibers (AcPULP). The chemical modification of pulp fibers with acetyl functionalities as depicted in Scheme 1 was carried out according to the following procedure:

Scheme 1. Schematic Representation of the Synthetic Approach Used for the Acetylation of Cellulosic Pulp, Carried out in THF at Room Temperature (RT)

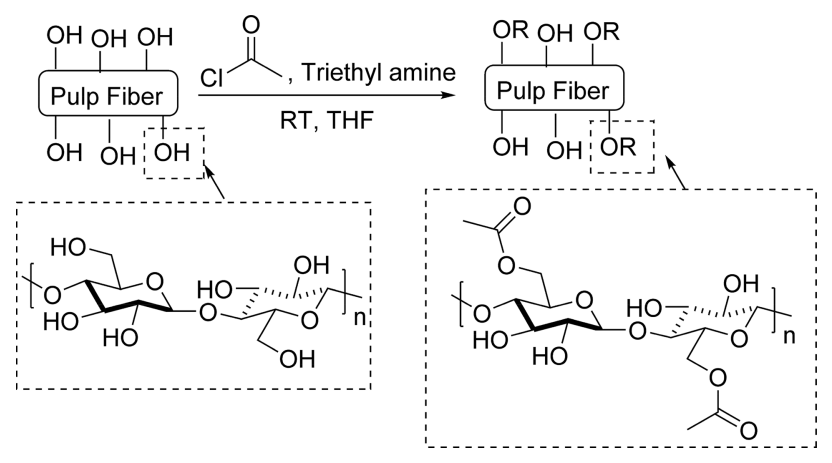

After the purification step, fibers were washed with three times the initial volume with water, solvent exchanged to five times the initial volume with acetone, and then solvent exchanged to five times the initial volume with THF.

Pulp fibers suspended in $1 \mathrm{~L}$ of THF together with TEA $(22.5 \mathrm{~g}$, $222.2 \mathrm{mmol}$ ) were placed in a $2 \mathrm{~L}$ round bottomed flask, equipped with magnetic stirrer. To this solution, acetyl chloride (14.5 g, 185.2 $\mathrm{mmol}$ ) was added dropwise. The reaction was allowed to proceed for 4 $\mathrm{h}$ at room temperature. Then, the acetylated pulp fibers were recovered by filtration and washed thoroughly by repeated dispersion filtration with THF, acetone, and then deionized water.

Fabrication of PULP (or AcPULP)/PCL Biocomposites. Prior to extrusion, PCL in powder form and varying the amount (from 0 to 20 wt $\%$ in dry content) of unmodified (PULP, $22 \%$ in dry content) or acetylated pulp fibers (AcPULP, $21 \%$ in dry content) were manually premixed prior to the "wet feeding" approach. Reference samples from the conventional "dry feeding" were prepared for the $20 \mathrm{wt} \%$ PULP or AcPULP/PCL biocomposite by drying each material for $8 \mathrm{~h}$ at $30{ }^{\circ} \mathrm{C}$ in a vacuum oven, prior to extrusion. Pulp fibers were dispersed in the PCL matrix by melt-blending using a DSM corotating twin-screw microcompounder (DSM, Holland, Explore, $5 \mathrm{cc}$ ) working at $120{ }^{\circ} \mathrm{C}$ first at $30 \mathrm{rpm}$ for $5 \mathrm{~min}$ for the feeding and then at $100 \mathrm{rpm}$ for about $15 \mathrm{~min}$, until the screw force value recorded during the processing reached a plateau. This was assumed to represent complete evaporation of the water during the processing. After compounding, 
and according to the ISO 527-2 standard, dumbbell (1BA) and rectangular specimens $(60 \times 10 \times 1 \mathrm{~mm})$, respectively, for the tensile testing and dynamical mechanical analysis (DMA), were prepared by injection molding using a HAAKE MiniJet-Pro (Thermo Fisher Scientific) with an injection pressure of $1000 \mathrm{bar}$, an oven temperature of $120{ }^{\circ} \mathrm{C}$, and mold temperature set at $40{ }^{\circ} \mathrm{C}$. The composition of different material melts processed, their initial water content, processing time, and acronym are reported in the Supporting Information, Table S1.

Characterization of Bio(nano)composites. Attenuated total reflectance-Fourier transform infrared (ATR-FTIR) was performed on a PerkinElmer Spectrum 2000 equipped with a MKII Golden Gate, Single Reflection ATR system from Specac Ltd., London, U.K. Spectra of unmodified and acetylated fibers were the average of 32 scans and were normalized with respect to the region from 2300 to $1900 \mathrm{~cm}^{-1}$ ascribed to the ATR crystal absorption region.

The morphology of the fibers and biocomposites was analyzed by wide-angle $\mathrm{x}$-ray diffraction (WAXD), scanning electron microscopy (SEM), and X-ray microtomography.

WAXD diffractograms were collected utilizing an ARL X'TRA powder diffractometer from Thermo Fisher Scientific Inc., USA, operated at $40 \mathrm{kV}$ and $40 \mathrm{~mA}$. The samples were scanned at $2 \mathrm{q}$ angular range $10-60^{\circ}$ and the increment was $0.02^{\circ}$. The plotted diffractograms are represented from $2 \mathrm{q}$ angular range $10-40^{\circ}$ as there was no useful information in the range of $40-60^{\circ}$.

High resolution scanning electron micrographs were taken by using a Hitachi SEM S-4800 (Japan) with an accelerating voltage of $1 \mathrm{kV}$. For the pulp, SEM samples were prepared from a water dispersion (0.05 wt \%) or before the processing or recovered from the biocomposites after Soxlhet extraction from chloroform and redispersed in water suspension, dried at room temperature prior observation, and finally $\mathrm{Pt} / \mathrm{Pd}(60 / 40)$ sputtered for $20 \mathrm{~s}$ at a current of $80 \mu \mathrm{A}$ using a Cressington 208HR sputter coater prior to imaging.

$\mathrm{X}$-ray microtomography was carried out on samples cut out from the injected dumbbell specimens by using an Xradia MicroXCT-200. Scanning conditions for the X-ray source were voltage $45 \mathrm{kV}$, power 4 $\mathrm{W}$, current $88 \mu \mathrm{A}$. Number of projections was 1105, exposition time $15 \mathrm{~s} /$ projection. A distance from the detector and X-ray source was 7.5 $\mathrm{mm}$ and $30 \mathrm{~mm}$, respectively. Used magnification was $20 \times$, pixel resolution was $1.0865 \mu \mathrm{m}$, and an analyzed volume of $1 \mathrm{~mm}$ diameter and $1 \mathrm{~mm}$ length was used.

Mean fiber length and fiber length distribution of original fibers, acetylated-fibers, as well as of extracted fibers from biocomposites were evaluated by means of a MorFi Compact fiber analyzer from TECHPAP (France). For this purpose, a dilute suspension of fibers $(25 \mathrm{mg} / \mathrm{L})$ was analyzed using an optics and flow cell measurement. Data of more than 3000 fibers were treated and the mean fiber length, mean weighted length, and the fiber length distribution were obtained. For the analysis of reinforcing fibers, biocomposites at $20 \mathrm{wt} \%$ fiber content were first submitted to Soxhlet extraction in chloroform for 8 h.

Thermogravimetrical analyses (TGA) were performed using a Mettler Toledo instrument, calibrated with indium, under air and nitrogen flow, from ambient temperature to $800{ }^{\circ} \mathrm{C}$ at a heating rate of $10{ }^{\circ} \mathrm{C} / \mathrm{min}$.

Thermal characterizations of the biocomposites were carried out using DSC by means of a Mettler Toledo TGA/DSC1 apparatus. A heating/cooling/heating procedure was applied over a temperature range from -80 to $180{ }^{\circ} \mathrm{C}$ at $10{ }^{\circ} \mathrm{C} / \mathrm{min}$. The glass-transition temperature $\left(T_{\mathrm{g}}\right)$, melting temperature $\left(T_{\mathrm{m}}\right)$ and melting enthalpy $\left(\Delta H_{\mathrm{m}}\right)$ were determined from the second heating.

Tensile tests and DMA of the resulting PCL-based biocomposites were performed on samples conditioned for $100 \mathrm{~h}$ at $23{ }^{\circ} \mathrm{C}$ and $50 \%$ $\mathrm{RH}$. The tensile tests were carried out using a Single Column Table Top Instron 5944 tensile microtester 5944 with a load force of $2 \mathrm{kN}$ and a deformation rate of $100 \%(30 \mathrm{~mm} / \mathrm{min})$, according to ASTM D638-14. Seven replicates were performed for each biocomposite formulation and data scattering was in the range of the 5-9\%. The DMA characterization was carried out using a Q800 DMA apparatus from TA Instruments in three-point bending mode, according to
ASTM D5023-07. Three replicates were performed for each biocomposite formulation and data scattering was below the $5 \%$. The DMA measurements were carried out at a constant frequency (1 $\mathrm{Hz}$ ), amplitude of $40 \mu \mathrm{m}$, a temperature range from -80 to $40^{\circ} \mathrm{C}$, and with a heating rate of $2{ }^{\circ} \mathrm{C} / \mathrm{min}$.

Size exclusion chromatography (SEC) was performed in $\mathrm{CHCl}_{3}$ (Fischer Scientific, HPLC grade) used as the eluent at a flow rate of $1.0 \mathrm{~mL} / \mathrm{min}$, and the injection volume was $50 \mathrm{~mL}$. The apparatus consisted of a Waters 717 Plus autosampler and a Waters (model 510) solvent pump equipped with a PL-ELS 1000 light scattering detector and three PL gel $10 \mathrm{~mm}$ mixed B columns $(3007.5 \mathrm{~mm})$ from Polymer Laboratories. Narrow molar mass polystyrene standards were used for calibration. The data were processed with Millennium software version 3.20 .

Nuclear magnetic resonance (NMR) (solution state ${ }^{1} \mathrm{H}-$ and ${ }^{13} \mathrm{C}-$ ) was recorded at room temperature on a Bruker Avance III HD $400 \mathrm{MHz}$ instrument with a BBFO probe equipped with a Z-gradient coil for structural analysis. Data were processed with MestreNova (Mestrelab Research) using $90^{\circ}$ shifted square sine-bell apodization window; baseline and phase correction was applied in both directions. Cross-polarization magic angle spinning carbon-13 nuclear magnetic resonance (CP/MAS ${ }^{13} \mathrm{C}$ NMR) spectra were recorded in a Bruker Avance III AQS $400 \mathrm{SB}$ instrument operating at $9.4 \mathrm{~T}$. Details are reported in the Supporting Information.

\section{RESULTS AND DISCUSSION}

Fiber Modification. Cellulose was modified up to a low cellulose degree of substitution (DS) in order to avoid decreased cellulose crystallinity. Figure 1A shows the ATR-

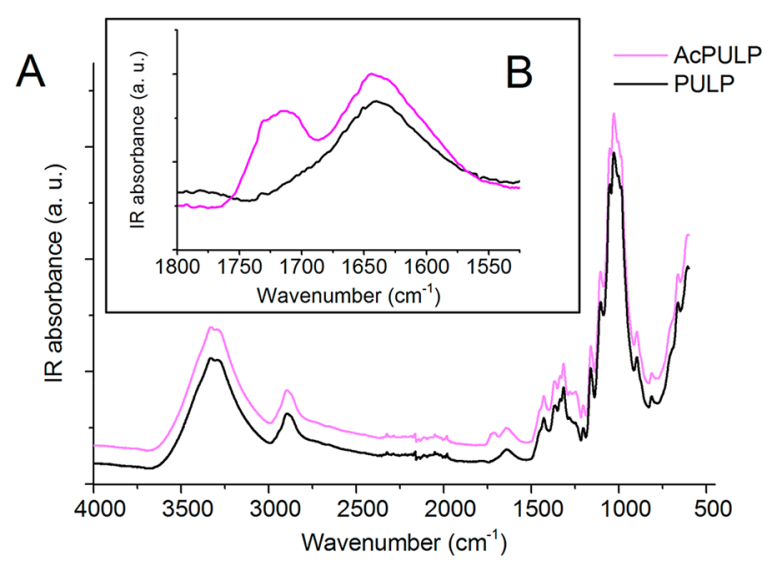

Figure 1. ATR-FTIR spectra of unmodified pulp and acetylated pulp (a) and its magnification in the region of ester stretch at $1730 \mathrm{~cm}^{-1}$.

FTIR spectra of unmodified pulp fibers and acetylated fibers. The emergence of a new peak at $1730 \mathrm{~cm}^{-1}$ suggests the successful surface modification, as evident from the region magnified in Figure 1B.

$\mathrm{CP} / \mathrm{MAS}$ solid state ${ }^{13} \mathrm{C}$ NMR data are presented in Figure 2 for the neat pulp and acetylated pulp to securely confirm the acetylation of the pulp fiber. In both neat pulp and acetylated pulp, peaks attributed to cellulose fiber anhydrous glucose unit (AHG) backbone are prevalent. Consistent with previous reports, carbons 2,3 , and 5 are located at $70-75$ ppm, whereas the $\mathrm{C} 1$ appears at $110 \mathrm{ppm}$, and finally $\mathrm{C} 4$ and $\mathrm{C} 6$ are found at 85 and 66 ppm, respectively (in Figure 2A). ${ }^{29,30}$ In the C4 and C6 separate signals can be seen. Consistent with modification via esterification, acetylated fibers show a methyl peak at 20.5 ppm (in Figure 2B) and a carbonyl $(\mathrm{C}=\mathrm{O})$ peak at $173 \mathrm{ppm}$. Cellulose crystallinity was determined as 45.6 and $45.5 \%$ for unmodified and acetylated fibers confirming that modification 


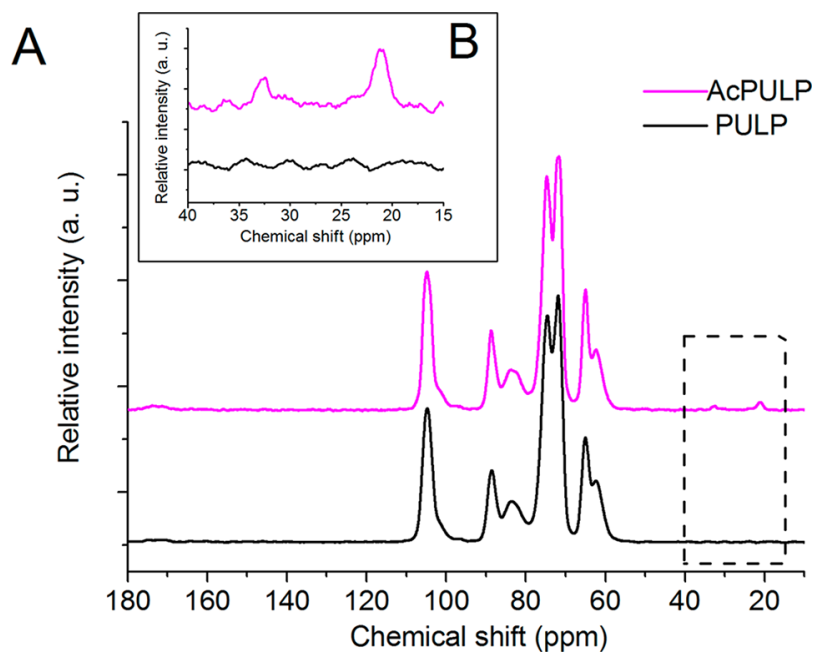

Figure 2. $\mathrm{CP} / \mathrm{MAS}{ }^{13} \mathrm{C}$ NMR spectra in the region of $120-40 \mathrm{ppm}$ for the acetylated pulp AcPULP and the unmodified PULP (A), and its magnification in the region of the methyl peak (B).

did not adversely affect cellulose crystallinity. The average bulk DS and surface DS assessed by CP/MAS ${ }^{13} \mathrm{C}$ NMR was 0.037 and 0.068 . It is worth noting here that the assessment of the surface DS is based on the geometry of a cellulose nanofibrils rather than for a pulp fiber, implying a considerably higher DS for the pulp surfaces. XRD diffractograms of neat unmodified and acetylated fibers (in Figure 3 ) showed cellulose $2 \Theta$ peaks at

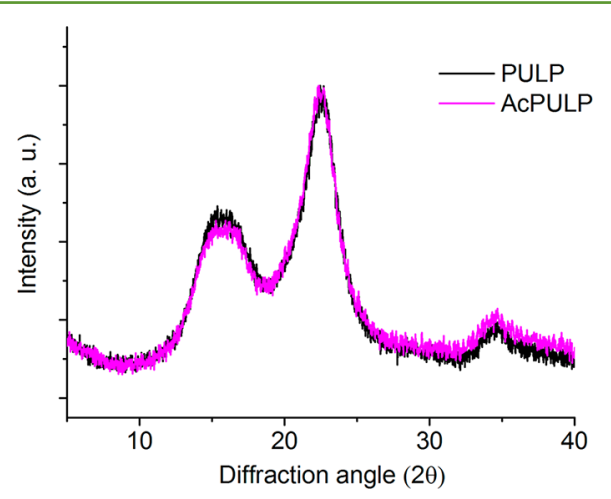

Figure 3. XRD diffractograms of unmodified (PULP) and acetylated pulp (AcPULP).

$14.6^{\circ}, 16.3^{\circ}, 20.3^{\circ}$ and $34^{\circ}$ related to $101,10 \overline{1}, 002$, and 004 planes, ${ }^{31}$ and confirming that the acetylation was carried out preserving the crystallinity of the pristine pulp.

Calculated crystallinity indexes from the XRD diffractograms are tabulated to be $47 \%$ for unmodified pulp fibers and $48 \%$ for acetylated pulp fibers, respectively, in agreement with the crystallinity assessed by $\mathrm{CP} / \mathrm{MAS}{ }^{13} \mathrm{C}$ NMR.

Fiber diameter for both the original pine pulp and the acetylated pine pulp was assessed by SEM, and shown in Figure 4 and in Table S3. Fiber length was evaluated by a fiber analyzer (Figure S1 and Table S3). Morphological studies comparing both neat pulp fibers and acetylated fibers pointed out no significant differences in overall structure, shape, or tabulated diameter. Both unmodified and modified fibers showed a slightly macroporous structure as revealed by the appearance of $2-3 \mu \mathrm{m}$ voids visible in both the micrographs in Figures $4 \mathrm{~B}$ and 4D. No significant difference in the fiber size distribution is observed (Figure S1 and Table S3). Mean

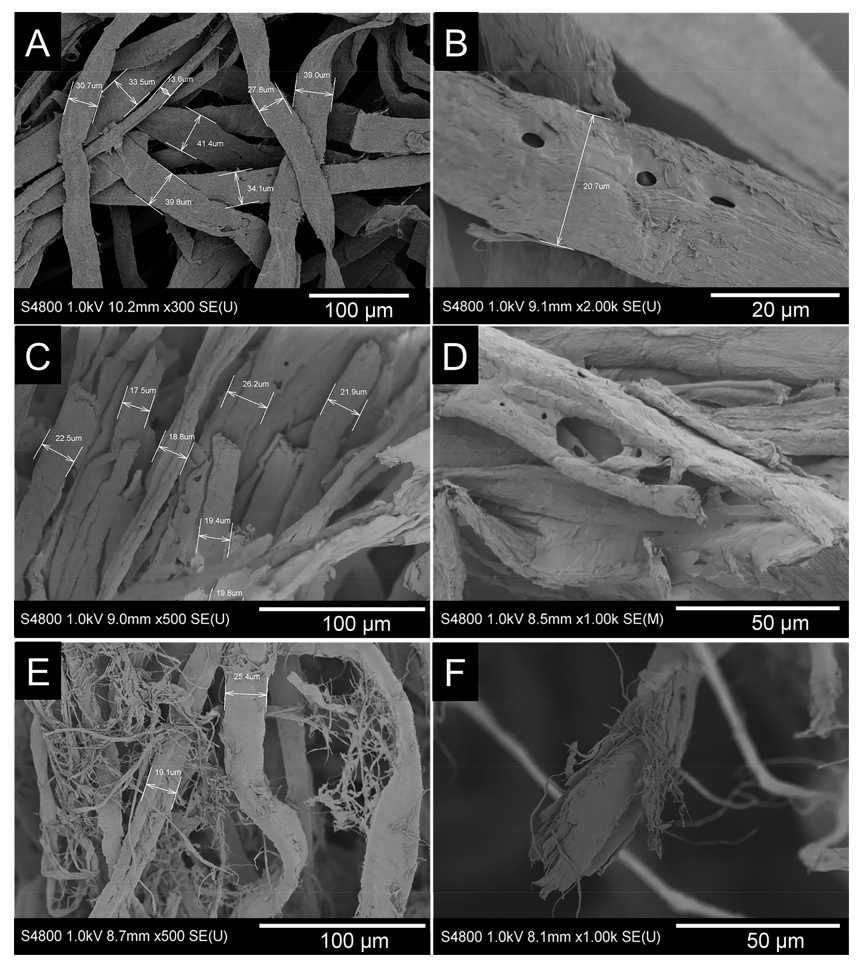

Figure 4. SEM micrographs of Kraft cellulosic pulp (PULP, A and B), after acetylation (AcPULP, C and D) and recovered after Soxhlet extraction in $\mathrm{CHCl}_{3}$ from the biocomposite $20 \mathrm{wt} \% \mathrm{AcPULP} / \mathrm{PCL}$ (E and F) at different magnifications. Scale bar: $100 \mu \mathrm{m}$ (A, C and E), 50 $\mu \mathrm{m}$ (D anf F), and $20 \mu \mathrm{m}$ (B).

arithmetic length of the original pine pulp was $1315 \mu \mathrm{m}$, compared to $1275 \mu \mathrm{m}$ for the acetylated fibers.

Cellulose thermal stability is important during melt extrusion of polymer composites. Thermogravimetrical analysis of the neat fibers was carried out and TGA results (in Figure S2) show that both neat pulp and acetylated pulp can be extruded at the selected processing temperature $\left(120^{\circ} \mathrm{C}\right)$. Indeed, both neat pulp and acetylated pulp have T5\%, (the temperature at which $5 \%$ of the material degrades) greater than $250{ }^{\circ} \mathrm{C}$.

Biocomposites. PCL composites were prepared using both wet and dry feeding of cellulose fibers. Composites showed no discoloration after compounding and injection molding. Only the dry fed 20\%AcPULP/PCL composites showed a slight brownish color, probably due to higher shear stress in the dry condition (Figure S3). Thermal stability of PCL composites was investigated using TGA under inert atmosphere; the TGA curves are shown in Figure S4. Briefly, the minor thermal stability with the increase of fiber content, in particular for the dry fed composites, is attributed to higher shear in the extruder as shown by the values reported in Table 1 , corresponding to the plateau values of the screw force (proportional to the torque, see also Figure S5) achieved during the processing for the different biocomposites compared to the neat PCL. Thus, biocomposites were made with varying amounts of water present during extrusion (from 5 to $50 \mathrm{wt} \%$ and varying the residence time in the microcompounder, ranging from 5 to 30 min. After extrusion, pulp fibers were recovered from biocomposites by Soxhlet extraction, and the solubilized PCL matrix was analyzed using SEC and ${ }^{1} \mathrm{HNMR}$ techniques. The results, in Figure 5 and Table S1, showed a small effect of either a large excess of water (50 wt \%) or a longer processing time (30 min, black curve in Figure 5A) on PCL $M w$ and dispersity 
Table 1. Main Results Recorded from the Dynamical Mechanical Analysis (DMA) and the Screw Force Recorded during the Processing for the Different Biocomposites. $T_{\mathrm{g}}$ was Determined as the Peak of the Loss Modulus G" , According to ASTM D5026-06. The Screw Force Recorded during the Melt Compounding. Standard Deviation Is <5\%

\begin{tabular}{lcccc}
\multicolumn{1}{c}{ sample } & $\begin{array}{c}G^{\prime} \text { at }-80{ }^{\circ} \mathrm{C} \\
{[\mathrm{GPa}]}\end{array}$ & $\begin{array}{c}\mathrm{G}^{\prime} \text { at } 20{ }^{\circ} \mathrm{C} \\
{[\mathrm{GPa}]}\end{array}$ & $\begin{array}{c}T_{\mathrm{g}} \\
{\left[{ }^{\circ} \mathrm{C}\right]}\end{array}$ & $\begin{array}{c}\text { force screw } \\
{[\mathrm{N}]}\end{array}$ \\
PCL & $4.2 \pm 0.1$ & $0.7 \pm 0.1$ & -44 & 1970 \\
20\% PULP/ & $8.8 \pm 0.4$ & $3.1 \pm 0.1$ & -48 & 2620 \\
PCL_WF & $7.6 \pm 0.4$ & $2.3 \pm 0.2$ & -47 & 3300 \\
$\begin{array}{l}\text { 20\% PULP/ } \\
\text { PCL_DF }\end{array}$ & & & & \\
20\% AcPULP/ & $9.3 \pm 0.3$ & $3.7 \pm 0.1$ & -47 & 1810 \\
PCL_WF & & & & \\
\hline
\end{tabular}
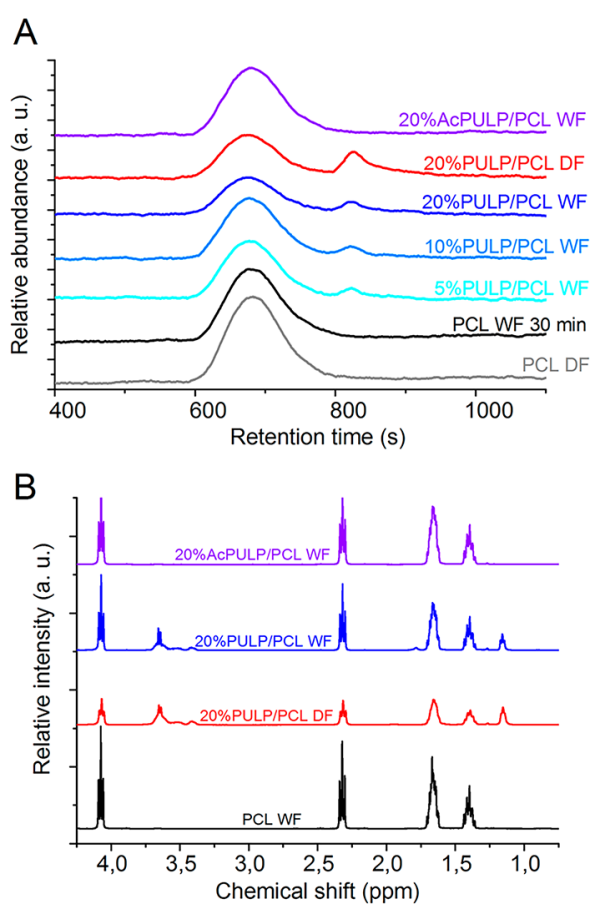

Figure 5. SEC curves (A) of the neat PCL before and after processing (50 wt \% of water for $30 \mathrm{~min}$ ) and the recovered soluble fractions from the Soxhlet extraction in $\mathrm{CHCl}_{3}$ of the wet fed biocomposites with the 5-20 wt \% (5-20\%PULP/PCL-WF), 20 wt \% dry fed biocomposites (20\%PULP/PCL-DF) and the 20 wt \% wet fed acetylated pulp biocomposites (20\%AcPULP/PCL-WF). ${ }^{1}$ HNMR spectra (B) of the neat PCL (PCL WF) and the recovered soluble fractions of the Soxhlet extraction from $\mathrm{CHCl}_{3}$ of the $20 \mathrm{wt} \%$ pulpbased biocomposites.

index, which not changed by more than $5 \%$ compared to neat PCL (gray curve in Figure 5A and black curve in Figure 5B). In the SEC curves (Figure 5A) a second population at higher retention time appears for the biocomposite with the unmodified pulp, with higher area at higher fiber content, confirming a thermal degradation of the polymer matrix due to the increased shear stress, as observed from TGA analysis. Surprisingly, the matrix recovered from the $20 \mathrm{wt} \%$ of AcPULP/PCL did not show any evidence of this second population in the SEC curve. The analysis of the ${ }^{1} \mathrm{HNMR}$ spectra, in Figure 5B is in agreement with the SEC analysis. Both dry and wet feeding with unmodified fibers resulted in an increased amount of PCL end groups (i.e., PCL-OH) as shown by the doublet at $3.25 \mathrm{ppm}$, attributed to a beta-scission mechanism caused by the high shear present in the system. ${ }^{32}$ In comparison, acetylated fibers show much less degradation due to the absence of PCL end groups suggesting an improved interaction and dispersion of acetylated cellulose fibers in the matrix. ${ }^{33}$ From DSC thermograms (Table S2), a very small effect of fiber loading is observed on PCL glass transition temperatures, melt temperatures and melting enthalpies $\left(\Delta H_{\mathrm{m}}\right)$, suggesting no nucleation effect of cellulose fibers in the already high crystalline polymer matrix. Only a slight increase in the melt enthalpy and the crystallinity in the dry fed biocomposite is observed, conceivably due to the presence of lower molecular weight PCL chains that can easily crystallize, according to the SEC and NMR analysis.

The effect of processing conditions on the mean fiber length and fiber length distribution was also investigated. Figure 6 shows the fiber length distributions of the fibers recovered from the biocomposites.
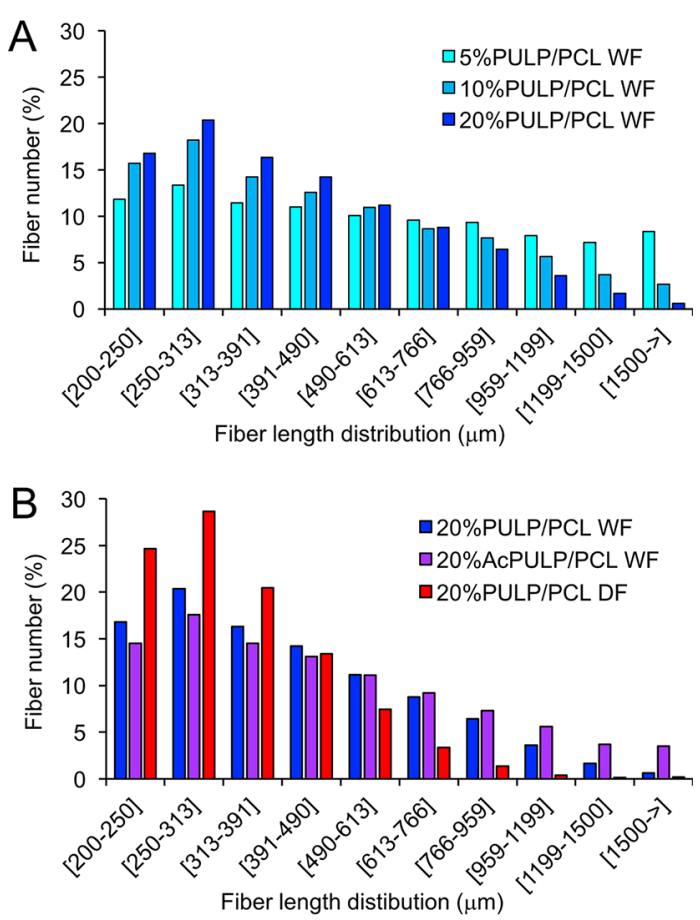

Figure 6. Fiber length distribution of the fibers recovered after Soxhlet extraction from $\mathrm{CHCl}_{3}$ of the different biocomposites PULP/PCL prepared by the wet feeding (WF) (A), and the different 20 wt \% biocomposites prepared with unmodified or acetylated fibers in wet or dry feeding (DF) (B).

The fiber length decreased considerably after the extrusion process. The mean fiber length in the biocomposites decreased with the fiber content. For the unmodified pulp-based biocomposites produced by wet feeding (Figure 6A and Table S3) the mean fiber lengths of 690,530 , and $465 \mu \mathrm{m}$ were assessed respectively for the biocomposites at 5,10 , and $20 \mathrm{wt} \%$ of pulp. This result reflects a milder shear stress during the melt compounding (Figure S5) due to the presence of water, showing as a decrease in the screw force (i.e., the viscosity of the system at the processing conditions), which increased gradually with the water evaporation. Moreover, the fiber shortening was more noticeable for dry feeding (Figure 6B and Table S3), with a final mean fiber length of $350 \mu \mathrm{m}$ for the $20 \mathrm{wt} \%$ biocomposite. Wet feeding is therefore suggested to preserve the fiber length during the extrusion process, resulting 
in an increase in the aspect ratio of the fibers (Table S3). The extrusion of acetylated fibers, with improved fiber-PCL compatibility, resulted in less fiber shortening with a mean fiber length of $550 \mu \mathrm{m}$ after extrusion and consequently an increase in the aspect ratio of the AcPULP with respect to the unmodified fibers (Table S3). Concurrent with these results, chemical affinity between components reduces shearing forces and prevents fiber length degradation.

The morphology of the fibers recovered after Soxhlet extraction from the biocomposites, suggested nanofibrillation effects. $^{23,25}$ In the current case, micro(nano)fibrillation was observed in the SEM micrographs of the acetylated fibers recovered from the 20\%AcPULP/PCL biocomposite (Figure $4 \mathrm{E}, \mathrm{F})$. Moreover, from the X-ray $3 \mathrm{D}$ reconstructions reported in Figure 7, significant changes in the morphology of the

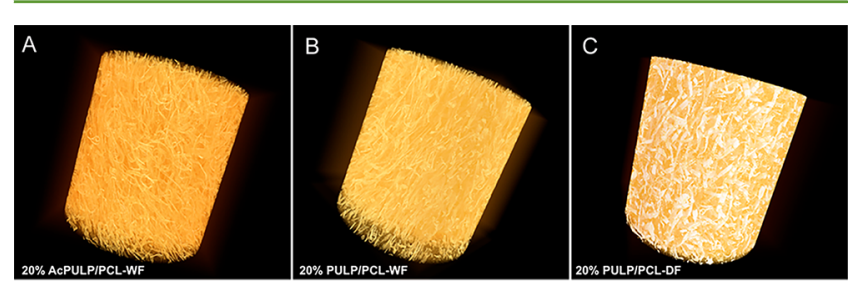

Figure 7. Rendered 3D volume reconstruction of X-ray microtomographs of the biocomposites. The volume size is of $1 \mathrm{~mm}$ diameter and $1 \mathrm{~mm}$ length; false color.

different biocomposites are distinguished. Large extent of aggregation was observed for dry feeding compared to wet feeding (Figures 7C,B, respectively). Conceivably wet feeding avoided the formation of strong fiber-fiber interactions (agglomeration) before and during processing, favoring the fiber dispersion and distribution within the PCL matrix. In addition, good fiber-matrix compatibility enhanced the fiber dispersion in the biocomposites. Chemical modification substantially affected the distribution of the pulp fibers and played a role in the in situ nanofibrillation of the fibers during processing. The best results were therefore obtained for wet feeding of acetylated fibers, with well-distributed fibers and significant nanofibrillation for the 20\%AcPULP/PCL biocomposites (Figure 7A). It can be also established that in situ nanofibrillation is mainly observed for wet-feeding of both unmodified and acetylated fibers.

Mechanical properties of the biocomposites were investigated using DMA and tensile tests. Their representative curves are shown in Figures 8 and 9, and in Tables 1 and 2, respectively. Figure $8 \mathrm{~A}$ presents the flexural storage and loss moduli curves for wet-fed biocomposites. Increased fiber content led to increased storage modulus both below and above the $T_{\mathrm{g}}$ of $\mathrm{PCL}$, located at $-44{ }^{\circ} \mathrm{C}$ (Figure $8 \mathrm{~A}$, graph below). At $5 \%$ pulp fiber content, a slight increase in the flexural storage modulus $\left(G^{\prime}\right)$ was observed. This increase was more substantial for biocomposites at 10 and $20 \mathrm{wt} \%$ fiber content, in particular above $T_{\mathrm{g}}$. Well-established micromechanics models based on the rule of mixtures expressions confirm that reinforcement effects from fibers of a given modulus are higher when the polymer modulus is lower. ${ }^{34}$ Although the $20 \mathrm{wt} \%$ composition was slightly stiffer than 10 wt $\%$, the increase was less than expected. One contributing factor is the reduced mean fiber length at higher fiber content (Figure 6). In Figure 8B, flexural storage and loss moduli curves are presented for three materials of the same fiber content $(20$
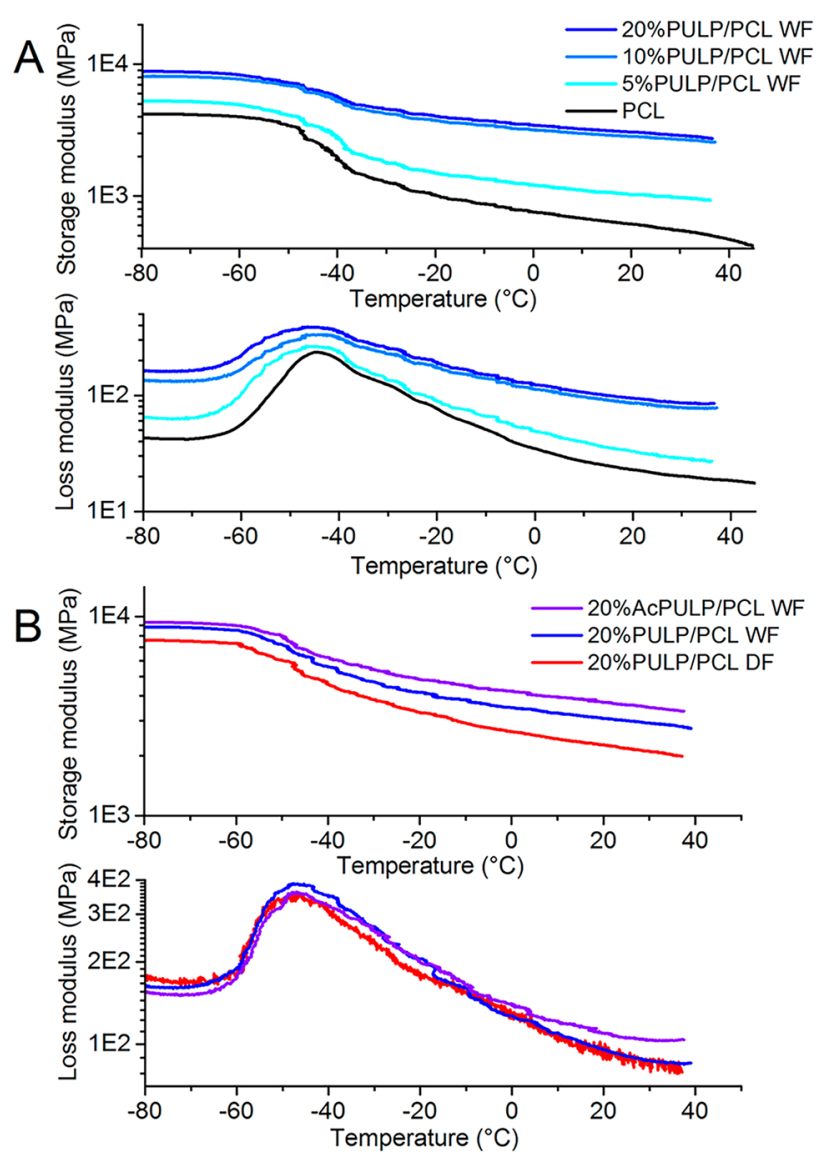

Figure 8. Log plot of the storage and loss moduli as a function of the temperature of the different biocomposites produced in wet feeding (A) and the $20 \%$ PULP-based biocomposites (B).

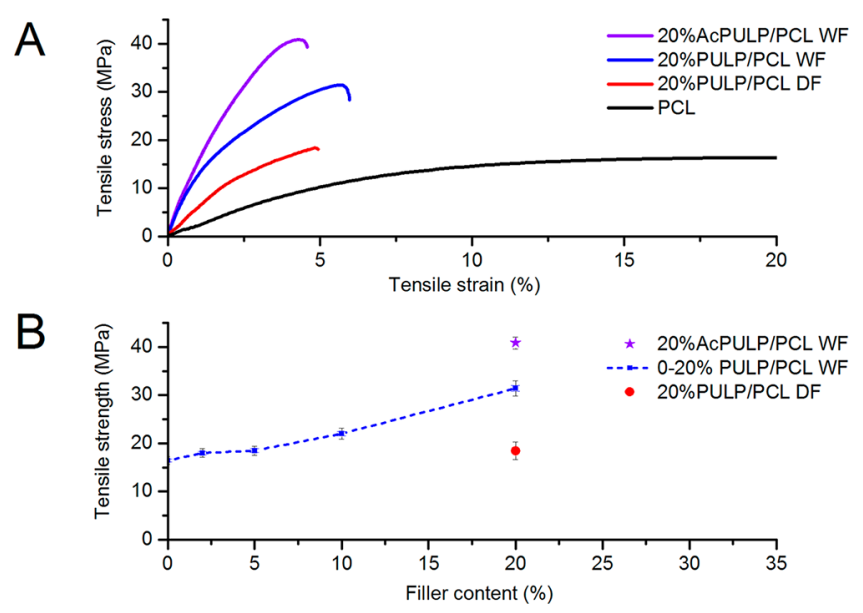

Figure 9. Stress-strain curves for the biocomposites with the $20 \mathrm{wt} \%$ of cellulosic filler compared with the neat PCL (A), and ultimate strength (B) as a function of the filler content for the different biocomposites.

wt \%). Again, the peaks of the loss modulus, related to the glass transition (Figure 8B, graph below) did not show significant shift with respect to fiber composition. It means there were no strong molecular scale effects on PCL mobility due to the fiber content. The reference material 20\%PULP/PCL prepared by dry feeding exhibited the lowest modulus. The main reasons are most likely the large extent of fiber agglomerates in the material and a reduced reinforcing efficiency for the decreased aspect 
Table 2. Main Mechanical Properties Recorded from the Tensile Tests Analysis for the Different Biocomposites. In Particular, Young's Modulus, Ultimate Strength and Elongation at Break. Standard Deviation Is $<5 \%$

\begin{tabular}{lccl}
\multicolumn{1}{c}{ sample } & $E_{\text {Young }}[\mathrm{GPa}]$ & $\sigma[\mathrm{MPa}]$ & $\varepsilon_{\text {break }}[\%]$ \\
PCL & $0.24 \pm 0.03$ & $16 \pm 0.24$ & $1510 \pm 43$ \\
5\% PULP/PCL_WF & $0.53 \pm 0.03$ & $19 \pm 0.34$ & $976 \pm 14$ \\
10\% PULP/PCL_WF & $0.87 \pm 0.04$ & $23 \pm 0.40$ & $709 \pm 8$ \\
20\% PULP/PCL_WF & $1.85 \pm 0.08$ & $31 \pm 0.71$ & $9 \pm 0.45$ \\
20\% PULP/PCL_DF & $0.59 \pm 0.06$ & $24 \pm 1.24$ & $5 \pm 1.20$ \\
20\% AcPULP/PCL_WF & $2.31 \pm 0.07$ & $41 \pm 0.80$ & $10 \pm 0.25$ \\
\hline
\end{tabular}

ratio (agglomerates and shortening) of the fibers into the material. Wet feeding (20\%PULP/PCL-WF) improved modulus substantially, since the fibers were better dispersed. The biocomposite from acetylated fibers (20\%AcPULP/PCL-WF) was the stiffest material. Therefore, the fiber-modification ameliorated the compatibility between fiber and matrix, and so the dispersion and the effective fiber aspect ratio, since the mean fiber length was also the highest for this composition. In a previous work, ${ }^{35}$ the storage moduli reported for the 20 wt \% flax fibers/PCL composites produced by melt processing was 4.5 GPa at $-70{ }^{\circ} \mathrm{C}$ and $1.7 \mathrm{GPa}$ at $20^{\circ} \mathrm{C}$, showing DMA curves translated to lower values in all the range of the temperature investigated.

Uniaxial tensile tests showed that biocomposites produced with 20 wt \% of fiber resulted in strong increase in Young's modulus and ultimate strength $(\sigma)$ (Figure 9 and Table 2). Young's modulus increased gradually from $0.24 \mathrm{GPa}$ for neat PCL to as high as $2.3 \mathrm{GPa}$ for the wet fed 20\%AcPULP/PCL. This is extremely significant since the low modulus at ambient conditions is a limitation of PCL. Data for strength are also encouraging. There was a gradual increase with fiber content, and the strength of the 20\%AcPULP/PCL-WF material was around $40 \mathrm{MPa}$. This strength value is at a strain of $4 \%$, where the neat PCL carries a stress of only around $10 \mathrm{MPa}$. The strong effect of degree of dispersion and effective aspect ratio on strength is apparent in Figure 9A,B. Most likely, fiber-matrix debonding is initiating failure, and this occurs at lower strains for compositions with more agglomerated fibers.

Both Young's modulus and ultimate tensile strength are thus increased with increasing fiber content. For comparison, the Young's modulus and the tensile strength previously reported for $20 \mathrm{wt} \%$ of flax fibers/PCL composites were $2.0 \mathrm{GPa}$ and 26 $\mathrm{MPa}$, respectively, ${ }^{35}$ and for $20 \mathrm{wt} \%$ hemp fibers/PCL were 0.3 $\mathrm{GPa}$ and $31 \mathrm{MPa}$, respectively. ${ }^{25}$

Strain to failure was reduced, as expected in a highly ductile polymer. The reason is the fiber-matrix debonding mechanism, originated by high local stress at the interface region. The wetfeeding reduces fiber agglomeration effects and mechanical properties are improved compared with dry feeding. In addition, acetylated fibers resulted in even higher modulus and strength due to combined effects from well-dispersed fibers and increased average fiber length.

\section{CONCLUSIONS}

In this work a facile and cost-effective method to produce high performance PCL-based biocomposites is proposed. The study highlights the importance of fiber modification effects in combination with a wet feeding compounding strategy compared to the traditional dry feeding one. Successful fiber modification with low DS was confirmed by FTIR and CP-
MAS solid state NMR. XRD diffraction, fiber morphology, and TGA studies showed that modification does not affect cellulose crystallinity or the fiber length, and that all fibers can be extruded at the selected temperature to allow the water evaporation during the melt processing $\left(120^{\circ} \mathrm{C}\right)$. In agreement with these results, all biocomposites produced were not discolored during processing. With the addition of $20 \mathrm{wt} \%$ cellulosic fibers, the Young's modulus increased from $240 \mathrm{MPa}$ (neat PCL) to $1850 \mathrm{MPa}$ for the biocomposites produced by using the wet feeding strategy. In comparison, biocomposites produced using dry-feeding had a Young's modulus of 690 $\mathrm{MPa}$. Wet feeding of acetylated cellulosic fibers (AcPULP) allowed even a greater increase, with an additional $46 \%$ and $248 \%$ increase of the ultimate strength and Young's modulus, when compared to wet feeding of the unmodified pulp, respectively. However, the gain in the values of the strength and Young's modulus due to the neat contribution of the chemical modification in the $20 \mathrm{wt} \%$ AcPULP/PCL is $37 \%$ (of the $46 \%$ ) and $26 \%$ (of the 248\%), respectively, compared to the biocomposite with the unmodified pulp. The results highlight the benefits of both the melt processing strategy (i.e., wet processing) as well as of the fiber surface modification, preventing aggregation and allowing for better dispersion of the cellulose fibers into the PCL matrix. As a consequence, cost-effective PULP/PCL-based biocomposites with outstanding mechanical performance are accomplished in the present study.

\section{ASSOCIATED CONTENT}

\section{Supporting Information}

The Supporting Information is available free of charge on the ACS Publications website at DOI: 10.1021/acssuschemeng.8b00551.

Solid state nuclear magnetic resonance details, fiber length distribution of the pulp before processing, TGA of the fibers before processing, visual aspect of the injection molded dumbbell specimens, TGA results of the biocomposites, screw force recorded during melt compounding, $\mathrm{CHCl}_{3} \mathrm{SEC}$ results (PDF)

\section{AUTHOR INFORMATION}

\section{Corresponding Authors}

*E-mail: giadalr@kth.se. Tel.: +46-8-790 8037. Fax: +468 207865.

*E-mail: blund@kth.se.

ORCID

Giada Lo Re: 0000-0001-8840-1172

\section{Funding}

Financial support from VINNOVA through the BiMaC Innovation Excellence Centre, funding from the Swedish Research Council Formas (2014-144) and for LB from SSF grant GMT14-0036 is gratefully acknowledged.

\section{Notes}

The authors declare no competing financial interest.

\section{ACKNOWLEDGMENTS}

The authors thank Assoc. Prof. Richard T. Olsson and Dr. Claudio Gioia for valuable discussions and inputs. 


\section{REFERENCES}

(1) Woodruff, M. A.; Hutmacher, D. W. The return of a forgotten polymer - Polycaprolactone in the 21st century. Prog. Polym. Sci. 2010, 35 (10), 1217-1256.

(2) Engelberg, I.; Kohn, J. Physico-mechanical properties of degradable polymers used in medical applications: a comparative study. Biomaterials 1991, 12 (3), 292-304.

(3) Spinella, S.; Lo Re, G.; Liu, B.; Dorgan, J.; Habibi, Y.; Leclere, P.; Raquez, J. M.; Dubois, P.; Gross, R. A. Polylactide/cellulose nanocrystal nanocomposites: Efficient routes for nanofiber modification and effects of nanofiber chemistry on PLA reinforcement. Polymer 2015, 65, 9-17.

(4) Lo Re, G.; Morreale, M.; Scaffaro, R.; La Mantia, F. P. Kenaffilled biodegradable composites: Rheological and mechanical behaviour. Polym. Int. 2012, 61 (10), 1542-1548.

(5) Scaffaro, R.; Morreale, M.; Lo Re, G.; La Mantia, F. P. Effect of the Processing Techniques on the Properties of Ecocomposites Based on Vegetable Oil-Derived Mater-Bi ${ }^{\circledR}$ and Wood Flour. J. Appl. Polym. Sci. 2009, 114, 2855-2863.

(6) Oksman, K.; Aitomäki, Y.; Mathew, A. P.; Siqueira, G.; Zhou, Q.; Butylina, S.; Hooshmand, S.; Tanpichai, S.; Zhou, X. Review of the recent developments in cellulose nanocomposite processing. Composites, Part A 2016, 83, 2-18.

(7) Lo Re, G.; Morreale, M.; Scaffaro, R.; La Mantia, F. P. Biodegradation paths of Mater- $\mathrm{Bi}$ ( $/$ kenaf biodegradable composites. J. Appl. Polym. Sci. 2013, 129 (6), 3198-3208.

(8) Scaffaro, R.; Morreale, M.; Lo Re, G.; La Mantia, F. P. Degradation of Mater-Bi®/Wood Flour Biocomposites in Active Sewage Sludge. Polym. Degrad. Stab. 2009, 94, 1220-1229.

(9) Moon, R. J.; Martini, A.; Nairn, J.; Simonsen, J.; Youngblood, J. Cellulose nanomaterials review: structure, properties and nanocomposites. Chem. Soc. Rev. 2011, 40 (7), 3941-3994.

(10) Eichhorn, S. J.; Dufresne, A.; Aranguren, M.; Marcovich, N. E.; Capadona, J. R.; Rowan, S. J.; Gindl, W. Review: current international research into cellulose nanofibers and nanocomposites. J. Mater. Sci. 2010, 45, 1-33.

(11) De la Orden, M. U.; Sánchez, C. G.; Quesada, M. G.; Urreaga, J. M. Effect of different coupling agents on the browning of cellulosepolypropylene composites during melt processing. Polym. Degrad. Stab. 2010, 95 (2), 201-206.

(12) Habibi, Y.; Goffin, A. L.; Schiltz, N.; Duquesne, E.; Dubois, P.; Dufresne, A. Bionanocomposites based on poly( $\varepsilon$-caprolactone)grafted cellulose nanocrystals by ring-opening polymerization. $J$. Mater. Chem. 2008, 18 (41), 5002-5010.

(13) Alvarez, V.; Mondragón, I.; Vázquez, A. Influence of chemical treatments on the interfacial adhesion between sisal fiber and different biodegradable polymers. Compos. Interfaces 2007, 14 (7-9), 605-616.

(14) Mukherjee, T.; Sani, M.; Kao, N.; Gupta, R. K.; Quazi, N.; Bhattacharya, S. Improved dispersion of cellulose microcrystals in polylactic acid (PLA) based composites applying surface acetylation. Chem. Eng. Sci. 2013, 101, 655-662.

(15) Spinella, S.; Maiorana, A.; Qian, Q.; Dawson, N. J.; Hepworth, V.; McCallum, S. A.; Gross, R. A.; Ganesh, M.; Singer, K. D. Concurrent cellulose hydrolysis and esterification to prepare a surfacemodified cellulose nanocrystal decorated with carboxylic acid moieties. ACS Sustainable Chem. Eng. 2016, 4 (3), 1538-1550.

(16) Spinella, S.; Lo Re, G.; Liu, B.; Dorgan, J.; Habibi, Y.; Leclere, P.; Gross, R. A.; Raquez, J.-M.; Dubois, P. Polylactide/cellulose nanocrystal nanocomposites: Efficient routes for nanofiber modification and effects of nanofiber chemistry on PLA reinforcement. Polymer 2015, 65, 9-17.

(17) Eyley, S.; Thielemans, W. Surface modification of cellulose nanocrystals. Nanoscale 2014, 6 (14), 7764-7779.

(18) Nishiyama, Y.; Langan, P.; Chanzy, H. Crystal structure and hydrogen-bonding system in cellulose $\mathrm{I} \beta$ from synchrotron $\mathrm{X}$-ray and neutron fiber diffraction. J. Am. Chem. Soc. 2002, 124 (31), 90749082.
(19) Newman, R. H. Carbon-13 NMR evidence for cocrystallization of cellulose as a mechanism for hornification of bleached kraft pulp. Cellulose 2004, 11 (1), 45-52.

(20) Idström, A.; Brelid, H.; Nydén, M.; Nordstierna, L. CP/MAS 13 C NMR study of pulp hornification using nanocrystalline cellulose as a model system. Carbohydr. Polym. 2013, 92 (1), 881-884.

(21) Karger-Kocsis, J.; Kmetty, Á.; Lendvai, L.; Drakopoulos, S. X.; Bárány, T. Water-assisted production of thermoplastic nanocomposites: a review. Materials 2015, 8 (1), 72-95.

(22) Rousseaux, D. D.; Sallem-Idrissi, N.; Baudouin, A. C.; Devaux, J.; Godard, P.; Marchand-Brynaert, J.; Sclavons, M. Water-assisted extrusion of polypropylene/clay nanocomposites: A comprehensive study. Polymer 2011, 52 (2), 443-451.

(23) Soulestin, J.; Quiévy, N.; Sclavons, M.; Devaux, J. Polyolefinsbiofiber composites: A new way for an industrial production. Polym. Eng. Sci. 2007, 47 (4), 467-476.

(24) Oksman, K.; Mathew, A. P.; Bondeson, D.; Kvien, I. Manufacturing process of cellulose whiskers/polylactic acid nanocomposites. Compos. Sci. Technol. 2006, 66 (15), 2776-2784.

(25) Beaugrand, J.; Berzin, F. Lignocellulosic fiber reinforced composites: influence of compounding conditions on defibrization and mechanical properties. J. Appl. Polym. Sci. 2013, 128 (2), $1227-$ 1238.

(26) Suzuki, K.; Homma, Y.; Igarashi, Y.; Okumura, H.; Semba, T.; Nakatsubo, F.; Yano, H. Investigation of the mechanism and effectiveness of cationic polymer as a compatibilizer in microfibrillated cellulose-reinforced polyolefins. Cellulose 2016, 23 (1), 623-635.

(27) Suzuki, K.; Okumura, H.; Kitagawa, K.; Sato, S.; Nakagaito, A. N.; Yano, H. Development of continuous process enabling nanofibrillation of pulp and melt compounding. Cellulose 2013, 20 (1), 201-210.

(28) Suzuki, K.; Sato, A.; Okumura, H.; Hashimoto, T.; Nakagaito, A. N.; Yano, H. Novel high-strength, micro fibrillated cellulose-reinforced polypropylene composites using a cationic polymer as compatibilizer. Cellulose 2014, 21 (1), 507-518.

(29) Larsson, P. T.; Wickholm, K.; Iversen, T. A CP/MAS ${ }^{13} \mathrm{C}$ NMR investigation of molecular ordering in celluloses. Carbohydr. Res. 1997, $302(1-2), 19-25$.

(30) Wickholm, K.; Larsson, P. T.; Iversen, T. Assignment of noncrystalline forms in cellulose I by CP/MAS 13 C NMR spectroscopy. Carbohydr. Res. 1998, 312, 123-129.

(31) Jandura, P.; Kokta, B. V.; Riedl, B. Fibrous long-chain organic acid cellulose esters and their characterization by diffuse reflectance FTIR spectroscopy, solid-state CP/MAS 13C-NMR, and X-ray diffraction. J. Appl. Polym. Sci. 2000, 78 (7), 1354-1365.

(32) Seo, K. S.; Cloyd, J. D. Kinetics of hydrolysis and thermal degradation of polyester melts. J. Appl. Polym. Sci. 1991, 42 (3), 845850 .

(33) Haque, R.; Saxena, M.; Shit, S. C.; Asokan, P. Fiber-matrix adhesion and properties evaluation of sisal polymer composite. Fibers Polym. 2015, 16 (1), 146-152.

(34) Affdl, J. C.; Kardos, J. L. The Halpin-The Halpin-Tsai equations: a review. Polym. Eng. Sci. 1976, 16 (5), 344-352.

(35) Leluk, K.; Kozlowski, M. Thermal and mechanical properties of flax-reinforced polycaprolactone composites. Fibers Polym. 2014, 15 (1), 108-116. 